REVISTA CIENCIAS BIOMÉDICAS

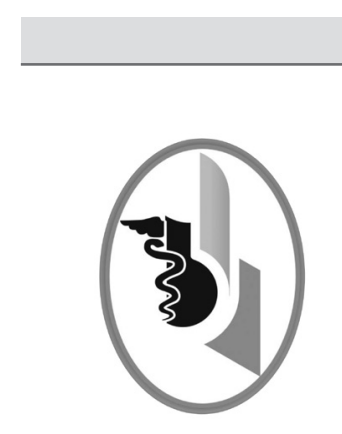

ARTÍCULOS ORIGINALES

\title{
CONSISTENCIA INTERNA DE LA ESCALA PARA DISCRIMINACIÓN MAYOR EN ESTUDIANTES DE MEDICINA
}

\author{
INTERNAL CONSISTENCY OF THE MAJOR DISCRIMINATION \\ SCALE IN MEDICINE STUDENTS
}

\author{
Campo-Arias Adalberto ${ }^{1}$ \\ Oviedo-Acevedo Heidi Celina ${ }^{2}$ \\ Herazo-Acevedo Edwin ${ }^{3}$ \\ Correspondencia: campoarias@comportamientohumano.org \\ Recibido para evaluación: febrero -14-2015. Aceptado para publicación: septiembre - 21 - 2016
}

\section{RESUMEN}

Introducción: la Escala para Discriminación Mayor (EDM) es un instrumento de nueve puntos para cuantificar las situaciones de discriminación -alguna vez en la vida-. En los Estados Unidos, la EDM mostró una alta confiabilidad, aunque, la confiabilidad se debe conocer en colectivos colombianos.

Objetivo: estimar la consistencia interna de la EDM en los estudiantes de Medicina de Bucaramanga-Colombia.

Materiales y métodos: un total de 361 estudiantes de Medicina completaron la EMD. La media para la edad de los participantes fue 20.1 años ( $D E=1.9$ ); el $59.6 \%$ de los estudiantes eran mujeres. La confiabilidad se calculó con el coeficiente de Kuder-Richardson (K-R) y la omega McDonald.

Resultados: la EMD mostró alta consistencia interna, coeficiente K-R de 0.76 y omega de McDonald de 0.85 .

Conclusión: la EMD es un instrumento con alta consistencia interna en estudiantes de Medicina de Bucaramanga-Colombia. Se puede usar este instrumento para la investigación de experiencias mayores de discriminación en estudiantes de Medicina. Rev. cienc.biomed.2016;7(2):234-239.

\section{PALABRAS CLAVE}

Discriminación social; Confiabilidad y validez; Estudiantes de Medicina; Estudios de validación.

1 Médico. Profesor Auxiliar. Grupo de investigación en Corazón y Diabetes. Programa de Medicina. Facultad de Ciencias de la Salud. Universidad del Magdalena. Santa Marta-Colombia.

2 Médica. Especialista en Psiquiatría. MSc E-learning. Grupo de Investigación del Comportamiento Humano. Bogotá-Colombia.

3 Médico. Especialista en Psiquiatría. Magíster en Bioética. MSc en Historia. PhD en Salud Pública (Universidad Nacional de Colombia). Grupo de Investigación del Comportamiento Humano Bogotá. Colombia. 


\section{SUMMARY}

Introduction: the Major Discrimination Scale (MDS) is a 9-item instrument to quantify situations of discrimination at some point in life. In the United States of America, the MDS showed high reliability, although, its reliability needs to be proven among Colombian people.

Objective: to estimate the reliability of MDS in Medicine students in BucaramangaColombia.

Material and methods: the MDS was completed with 361 Medicine students. The average age was 20.1 years $(S D=1.9)$, where the $59.6 \%$ were women. Reliability was calculated with Kuder-Richardson (K-R) coefficient and McDonald's omega.

Results: the MDS showed high internal consistency. The K-R coefficient was 0.76 and omega coefficient 0.85 .

Conclusion: the MDS presents a high internal consistency among Medicine students from Bucaramanga-Colombia. The MDS may use to measure major experiences of discrimination in Medicine Colombian students. Rev.cienc.biomed.2016;7(2):234-239.

\section{KEYWORDS}

Social discrimination; Reproducibility of results; Medical students; Validation studies.

\section{INTRODUCCIÓN}

El estigma, el prejuicio y la discriminación representan un problema dado que explican en parte la inequidad observada en el área de la salud (1). El estigma y la discriminación funcionan como estresores en las poblaciones que los afrontan, y deterioran el bienestar general, la salud física y mental (2).

Tradicionalmente, se han estudiado con mayor atención el estigma y la discriminación étnicoracial y de género $(3,4)$, y más recientemente, la medición de la identidad étnico-racial (5). No por ello dejan de ser frecuentes, y generan sufrimiento en las poblaciones, al igual que otro tipo de conductas discriminatorias: la discriminación relacionada con la edad, la presencia de discapacidad, por la orientación sexual o por aspectos relacionados con trastorno mental (6-8).

Parte de las limitaciones en la investigación sobre el impacto del estigma y la discriminación en los colectivos surgen ante la dificultad de contar con instrumentos de medición válidos y confiables para cuantificar la discriminación que puede sufrir un grupo de personas (perpetrador) y la discriminación percibida o sentida (víctima), y los distintos tipos o matices que puede tomar la discriminación $(9,10)$.

En consecuencia, existen muchas escalas para cuantificar el estigma y la discrimi- nación (auto o heterodiscriminación) por distintos motivos, tanto en las víctimas como en los perpetradores (11-13). William et al., diseñaron la EDM con el propósito de cuantificar el trato desigual y discriminatorio en diferentes contextos de la vida cotidiana: la ascendencia u origen nacional, el sexo, la raza o etnia, la edad, la religión, la estatura o el peso, el color de piel, la orientación sexual, el nivel de educación, la cantidad de ingresos económicos, la presencia de discapacidad o limitación física y/o cualquier otra causa (14). Esta medición de discriminación -alguna vez en la vida- se utilizó en un estudio epidemiológico con la participación de más de 3000 personas en los Estados Unidos con aceptable validez (15).

El desempeño psicométrico de los instrumentos varía según las características y los ámbitos de uso (16). Asimismo, según las bases teóricas que se tomaron, la construcción y las formas posibles para el diligenciamiento se pueden llevar a cabo con algunas mediciones de la validez y la confiabilidad (17). Una medida de uso frecuente para estimar en forma cuantitativa, parcial, $y$, simultáneamente, la validez y la confiabilidad de una escala de medición para las experiencias de discriminación es el cálculo de la consistencia interna (18). Las pruebas estadísticas que se usan para este propósito son los coeficientes de alfa de Cronbach (19) y omega de McDonald (20). 
La aproximación a la validez y la confiabilidad de la EDM, mediante el cálculo de la consistencia interna, permitirá el empleo de la misma en próximas investigaciones orientadas a conocer la frecuencia y las variables asociadas a discriminación en estudiantes de Medicina de Colombia. El propósito de este estudio fue cuantificar la consistencia interna de la EDM en un grupo de estudiantes de Medicina de una universidad de Bucaramanga, Colombia.

\section{MATERIALES Y MÉTODOS}

Se diseñó un estudio de validación sin criterio de referencia y con una sola aplicación de la escala para evaluar la consistencia interna. Un comité de ética en investigación de una universidad revisó y aprobó la ejecución del proyecto dado, que representaba un riesgo mínimo para los estudiantes puesto que no se llevaron a cabo intervenciones farmacológicas o psicosociales, según las normas colombianas para la investigación en salud (21).

Una muestra de voluntarios formada por 361 estudiantes de Medicina completó la información demográfica básica y la EDM. Se incluyeron estudiantes mayores de edad (18 años o más de edad; la media fue 20.1; $D E=1.9$ ). El $59.7 \%$ de los participantes estuvo representado por mujeres. Los estudiantes cursaban entre primero y noveno semestre; el $55.1 \%$ semestres del ciclo de materias básicas (primero y quinto semestre) y residían en todos los estratos socioeconómicos existentes en Colombia, entre I y VI; el $89.8 \%$ informaron residencia entre IV y VI semestre.

El instrumento a evaluar, la EDS, consta de nueve puntos que indagan la experiencia de discriminación por diferentes motivos -alguna vez en la vida-. Aquellos que fueron mencionados en la introducción. Cada pregunta ofrece dos opciones de respuesta: afirmativa o negativa (14).

Es importante tener presente que para esta investigación no se llevó a cabo un proceso de retro-traducción, dado que el instrumento se validó originalmente tanto en inglés como en español. No obstante, se hicieron algunos ajustes menores en la traducción disponible para hacerlo más apropiado para el español de uso habitual en Colombia (22, 23). La versión que se aplicó se presenta en la Tabla No 1.

Para conocer la consistencia interna se calculó el coeficiente de Kuder-Richardson (24). Este coeficiente es equivalente al alfa de Cronbach, sin embargo, el cálculo se indica cuando las opciones de la escala de medición ofrecen un patrón de respuesta dicotómico. El coeficiente alfa de Cronbach se usa en los casos en que las opciones de respuestas son politómicas u ordinales (19).

Dado que el cálculo del alfa de Cronbach parte del supuesto de que pocas veces se observa que los puntos que hacen parte de la escala de medición son tau-equivalentes, es decir, muestran pesos o coeficientes estadísticamente similares, en la actualidad se recomienda el cálculo de la omega de McDonald como medida adicional de confiabilidad. Este coeficiente es una mejor medida de la consistencia interna, que se tiende a subestimar con el incumplimiento de la tauequivalencia (20). La estimación de la omega de McDonald se llevó a cabo en forma manual puesto que los paquetes estadísticos no cuentan con comandos para ese proceso. Los otros cálculos se realizaron en el paquete estadístico STATA (25).

\section{RESULTADOS}

La consistencia interna de EDS fue alta, mostró un coeficiente de Kuder-Richardson de 0.76 y un coeficiente de McDonald de 0.85 . Ver patrón de respuesta en la Tabla $\mathrm{N}^{\circ} 2$. Un total de 110 estudiantes (30.5\%) respondió de forma afirmativa alguno de los puntos.

\section{DISCUSIÓN}

La presente investigación muestra que la EDM posee alta consistencia interna en estudiantes de Medicina de una universidad de Bucaramanga. Los hallazgos son consistentes con los estudios precedentes que mostraron que la EDM es un instrumento práctico y útil para la estimación de discriminación mayor (14). 


\begin{tabular}{|c|c|c|}
\hline \multicolumn{3}{|l|}{$\begin{array}{l}\text { TABLA No } 1 . \\
\text { ESCALA PARA DISCRIMINACIÓN MAYOR (EDM) }\end{array}$} \\
\hline PUNTOS & $\begin{array}{l}\text { Yes } \\
\text { (Sí) }\end{array}$ & $\begin{array}{c}\text { No } \\
\text { (No) }\end{array}$ \\
\hline $\begin{array}{l}\text { At any time in your life, have you ever been unfairly fired? } \\
\text { (¿Alguna vez en la vida, a usted le despidieron injustamente?) }\end{array}$ & & \\
\hline $\begin{array}{l}\text { For unfair reasons, have you ever not been hired for a job? } \\
\text { (¿Por razones injustas le negaron empleo?) }\end{array}$ & & \\
\hline $\begin{array}{l}\text { Have you ever been unfairly denied a promotion? } \\
\text { (¿Por razones injustas le negaron un ascenso laboral?) }\end{array}$ & & \\
\hline $\begin{array}{l}\text { Have you ever been unfairly stopped, searched, questioned, physically threatened } \\
\text { or abused by the police? } \\
\text { (¿Alguna vez la policía, le paró, registró físicamente, interrogó, amenazó o } \\
\text { maltrato injustamente?) }\end{array}$ & & \\
\hline $\begin{array}{l}\text { Have you ever been unfairly discouraged by a teacher or advisor from continuing } \\
\text { your education? } \\
\text { (¿Alguna vez algún docente o profesor le desanimó injustamente a continuar su } \\
\text { educación?) }\end{array}$ & & \\
\hline $\begin{array}{l}\text { Have you ever been unfairly prevented from moving into a neighborhood because } \\
\text { the landlord or a realtor refused to sell or rent you a house or apartment? } \\
\text { (¿Alguna vez injustamente le impidieron mudarse a un barrio porque el propietario } \\
\text { o arrendador se negaron a venderle o alquilarle?) }\end{array}$ & & \\
\hline $\begin{array}{l}\text { Have you ever moved into a neighborhood where neighbors made life difficult for } \\
\text { you or your family? } \\
\text { (¿Alguna vez se mudó a otro barrio porque los vecinos le hicieron la vida difícil a } \\
\text { usted o a su familia?) }\end{array}$ & & \\
\hline $\begin{array}{l}\text { Have you ever been unfairly denied a bank loan? } \\
\text { (¿A usted le han negado injustamente un crédito o préstamo bancario?) }\end{array}$ & & \\
\hline $\begin{array}{l}\text { Have you ever received service from someone such as a plumber or car mechanic } \\
\text { that was worse than what other people get? } \\
\text { (¿Ha recibido algún servicio de alguien, como plomero o conductor, que era peor } \\
\text { que el que otras personas recibieron?) }\end{array}$ & & \\
\hline
\end{tabular}

\begin{tabular}{|l|c|c|c|}
\hline \multicolumn{4}{|c|}{ TABLA No 2. } \\
\hline \multicolumn{4}{|c|}{ FRECUENCIA DE RESPUESTA PARA LOS PUNTOS DE EDM } \\
\hline Punto & $\mathrm{f}$ & $\%$ & K-R* \\
\hline Despido & 24 & 6.6 & 0.7 \\
\hline Empleo & 18 & 5.0 & 0.7 \\
\hline Ascenso laboral & 15 & 4.2 & 0.7 \\
\hline Policía & 35 & 9.7 & 0.7 \\
\hline Profesor & 63 & 17.5 & 0.8 \\
\hline Mudanza & 13 & 3.6 & 0.7 \\
\hline Vida difícil & 18 & 5.0 & 0.7 \\
\hline Préstamo bancario & 14 & 3.9 & 0.7 \\
\hline Servicio & 37 & 10.2 & 0.8 \\
\hline
\end{tabular}

* Kuder-Richardson con la omisión del punto. 
En condiciones ideales se espera que la consistencia interna de una escala de medición en salud se encuentre entre 0.8 y 0.9 , como en el presente estudio $(18,26)$. En el estudio precedente no se estimó la omega de McDonald, pero, el hallazgo de la investigación corrobora la alta consistencia interna de la medición. El incumplimiento del principio de tau-equivalencia para los puntos de la escala, dado que los valores de Kuder-Richardson y McDonald fueron disimiles, sugiere que es preferible informar la consistencia con la prueba de McDonald por la distribución de frecuencia de las respuestas para cada punto que, es sustancialmente distinta, como se observa en la Tabla $N^{\circ} 2(18,20)$.

Todos los aspectos relacionados con el estigma y discriminación ganan importancia día a día como fenómenos de salud pública, puesto que están altamente implicados en el bienestar colectivo $(6-8,27)$. Dada la diversidad que caracteriza a Colombia es necesario conocer el desempeño de este tipo de mediciones y contar con instrumentos que permitan una evaluación válida y confiable en diferentes contextos y hacer posible comparaciones aceptables entre los distintos te- rritorios que integran este país $(22,23,28)$. La validación de instrumentos de medición en salud es un proceso de constante revisión y adaptación en los diferentes grupos de poblaciones y contextos, sin cambiar los aspectos esenciales de la medición $(29,30)$.

Como conclusión, la EDM es un instrumento que presenta alta consistencia interna y que sugiere un grado aceptable de validez y confiabilidad en estudiantes de Medicina de una universidad de Bucaramanga-Colombia. Es necesario llevar a cabo este proceso de validación de la escala en diferentes colectivos del país.

\section{AGRADECIMIENTOS}

El Instituto de Investigación del Comportamiento Humano (Human Behavioral Research Institute) Bogotá-Colombia.

CONFLICTO DE INTERESES: ninguno que declarar.

FINANCIACIÓN: instituto de Investigación del Comportamiento Humano (Human Behavioral Research Institute) Bogotá-Colombia.

\section{REFERENCIAS BIBLIOGRÁFICAS}

1. Hatzenbuehler ML, Phelan JC, Link BG. Stigma as a fundamental cause of population health inequalities. Am J Public health. 2013;103:813-21.

2. Chen J. Perceived discrimination and subjective well-being among rural-to-urban migrants in China. J Social Soc Welfare. 2013;40:131-56.

3. Paradies Y. A systematic review of empirical research on self-reported racism and health. Int J Epidemiol. 2006; 35: 888-901.

4. Pascoe EA, Smart L. Perceived discrimination and health: a meta-analytic review. Psychol Bull. 2009;135:531-54.

5. Helms JE. Some better practices for measuring racial and ethnic identity constructs. J Counsel Psychol. 2007; 54:235-46.

6. Sartorius N, Gaetbel W, Cleveland H-R, Stuart H, Akiyama T, Arboleda-Flórez J, et al. WPA guidance on how to combat stigmatization of psychiatry and psychiatrists. World Psychiatry 2010;9:131-44.

7. Alvarez-Galvez J, Salvador-Carulla L. Perceived discrimination and self-rated health in Europe: Evidence from the European Social Survey (2010). PloS One. 2013; 8: e74252.

8. Goto J, Couto P, Bastos J. Revisão sistemática dos estudos epidemiológicos sobre discriminação interpessoal e saúde mental. Cad Saude Publica. 2013; 29: 445-459.

9. Krieger N. Methods for the scientific study of discrimination and health: an ecosocial approach. Am J Public Health. 2012;102:936-45.

10. Jones KP, Peddie CI, Gilrane VL, King EB, Gray AL. Not so subtle. A meta-analytic investigation of the correlates of subtle and overt discrimination. J Manag. 2013 (ahead of print).

11. Kressin NR, Raymond KL, Manze M. Perceptions of race/ethnicity-based discrimination: a review of measures and evaluation of their usefulness for the health care setting. J Health Care Poor Underserved. 2008; 19: 697-730.

12. Mora-Rios J, Bautista-Aguilar N, Natera G, Pedersen D. Adaptación cultural de instrumentos de medida sobre estigma y enfermedad mental en la Ciudad de México. Salud Mental. 2013;36:9-18. 
13. Grey JA, Robinson BBE, Coleman E, Bockting WO. A systematic review of instruments that measure attitudes toward homosexual men. J Sex Res. 2013; 50: 329-52.

14. Williams DR, Yu Y, Jackson JS, Anderson NB. Racial differences in physical and mental health socio-economic status, stress and discrimination. J Health Psychol. 1997; 2:335-51.

15. Kessler RC, Mickelson KD, Williams DR. The prevalence, distribution, and mental health correlates of perceived discrimination in the United States. J Health Soc Behav. $1999 ; 40: 208-30$.

16. Blacker D, Endicott J. Psychometric properties: concepts of reliability and validity. In: Handbook of psychiatric measures. Washington: American Psychiatric Association; 2002.

17. Sánchez R, Echeverry J. Validación de escalas de medición en salud. Rev Salud Publica. 2004;6:302-318.

18. Campo-Arias A, Oviedo HC. Propiedades psicométricas de una escala: la consistencia interna. Rev Salud Pública. 2008; 10: 831-839.

19. Cronbach J. Coefficient alpha and the internal structure of test. Psychometrika. 1951; 16: 297-334.

20. McDonald RP. Theoretical foundations of principal factor analysis and alpha factor analysis. Br J Math Stat Psychol. 1970;23:1-21.

21. Resolución 008430 por la cual se establecen las normas científicas, técnicas y administrativas para la investigación en salud. Santa Fe de Bogotá: Ministerio de salud; 1993.

22. Sousa VM, Rojjanasrirat W. Translation, adaptation and validation of instruments or scales for use in cross-cultural health care research: a clear and user-friendly guideline. J Eval Clin Pract. 2011;17:268-74.

23. Clark MJ. Cross-cultural research: Challenge and competence. Int J Nurs Pract. 2012; 18 (Suppl. 2): 28-37.

24. Kuder GF, Richardson MV. The theory of the estimation of test reliability. Psychometrika. 1937; 2: 151-160.

25. STATA 9.0 for windows. College Station: StataCorp LP; 2005.

26. Oviedo HC, Campo-Arias A. Aproximación al uso del coeficiente alfa de Cronbach. Rev Colomb Psiquiatr. 2005; 34: 572-580.

27. Hunte $\mathrm{HE}$, King $\mathrm{K}$, Hicken $\mathrm{M}$, Lee $\mathrm{H}$, Lewis $\mathrm{TT}$. Interpersonal discrimination and depressive symptomatology: examination of several personality-related characteristics as potential confounders in a racial/ethnic heterogeneous adult sample. BMC Public Health. 2013; 13: 1084.

28. Fletcher RH, Fletcher SW. Clinical epidemiology. The essentials. Fourth edition. Philadelphia: Lippincott Williams \& Wilkins; 2005.

29. Cook DA, Beckman TJ. Current concepts in validity and reliability for psychometric instruments: Theory and application. Am J Med. 2006; 119: 166.e7-166.e16.

30. Sullivan GM. A primer on the validity of assessment instruments. J Grad Med Educ. 2011; 3: 119-120.

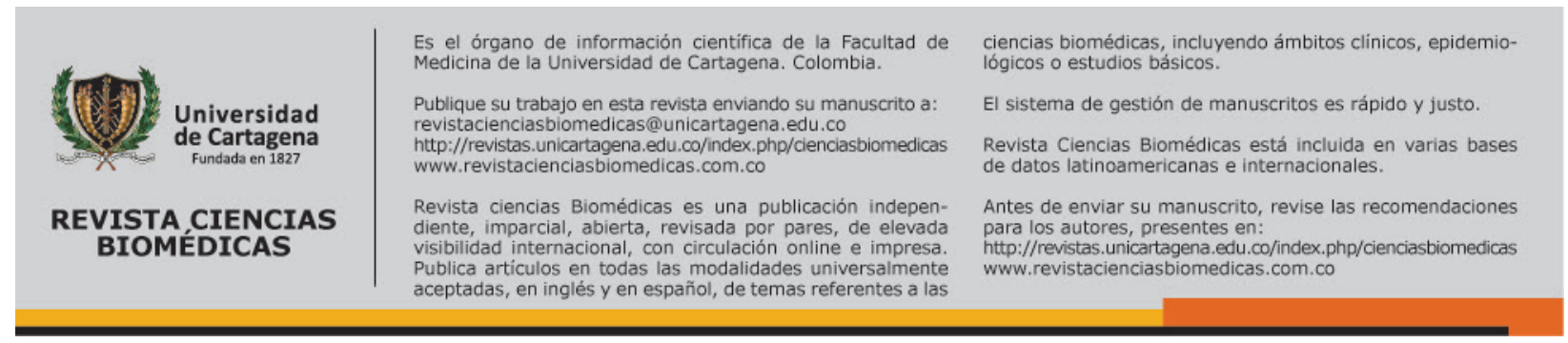

